\title{
Contrast-enhancement cardiac magnetic resonance imaging beyond the scope of viability
}

\author{
M. A. G. M. Olimulder • M. A. Galjee • J. van Es • \\ L. J. Wagenaar • C. von Birgelen
}

Published online: 3 March 2011

(C) The Author(s) 2011. This article is published with open access at Springerlink.com

\begin{abstract}
The clinical applications of cardiovascular magnetic resonance imaging with contrast enhancement are expanding. Besides the direct visualisation of viable and non-viable myocardium, this technique is increasingly used in a variety of cardiac disorders to determine the exact aetiology, guide proper treatment, and predict outcome and prognosis. In this review, we discuss the value of cardiovascular magnetic resonance imaging with contrast enhancement in a range of cardiac disorders, in which this technique may provide insights beyond the scope of myocardial viability.
\end{abstract}

Keywords Contrast enhancement - Cardiovascular magnetic resonance imaging . Cardiac involvement . Cardiomyopathy . Tissue characterisation

\section{Introduction}

Contrast-enhancement (CE) cardiovascular magnetic resonance (CMR) imaging is a technique that was initially developed to distinguish viable from non-viable myocardium following myocardial infarction (MI) [1-3]. In addition,

M. A. G. M. Olimulder • M. A. Galjee $(\bowtie) \cdot$ J. van Es •

L. J. Wagenaar $\cdot$ C. von Birgelen

Department of Cardiology, Thoraxcentrum Twente,

Medisch Spectrum Twente,

Haaksbergerstraat 55,

7513 ER, Enschede, the Netherlands

e-mail: M.Galjee@mst.nl

C. von Birgelen

MIRA-Institute for Biomedical Technology and Technical

Medicine, University of Twente,

Enschede, the Netherlands
CE-CMR is increasingly used for tissue characterisation in a broad spectrum of clinical applications, which are illustrated and discussed in this manuscript. Besides its ability to measure the size of myocardial scars or fibrosis, CE-CMR can determine the exact aetiology of disease, guide proper treatment, and predict outcome and prognosis in a variety of cardiac diseases; examples may be (non)-ischaemic cardiomyopathies, myocarditis, intracardiac masses, and myocardial involvement in systemic diseases.

\section{Brief technical background of CE-CMR}

The technique of CE-CMR imaging involves an intravenous injection of a contrast agent (e.g., gadolinium at a preferred dose of $0.2 \mathrm{mmol} / \mathrm{kg}$ body weight) followed by an ECG-gated T1-weight pulse sequence 10-15 min after the injection [4]. The timing of the image acquisition is of paramount importance, as too early image acquisition reduces the difference in contrast between normal and damaged myocardium (such as scar or fibrosis) because of an insufficient washout of contrast medium from the normal myocardium; too late image acquisition, on the other hand, may result in an excessive washout from damaged myocardial tissue that leads to an inferior signal-to-noise ratio [2]. The typical pulse sequence for CE-CMR imaging is a segmented T1-weighted inversion-recovery-prepared fast gradient-echo sequence. An inversion-recovery pulse is used to null the signal of normal myocardium in order to optimise the difference between normal and damaged myocardial areas (which still contain contrast medium) (Fig. 1).

The optimal inversion time depends on the contrast clearance from the normal myocardium which may show considerable inter-patient variability, depending on several factors such as the patient weight, and left ventricular (LV) or 




Fig. 1 Schematic figure for inversion time (TI) mapping. Following the ECG trigger, an inversion-recovery $(I R)$ pulse is applied. Before image acquisition, low-resolution TI scout images at mid-ventricular level with increasing TI (interval TI, $30 \mathrm{~ms}$ ) are performed. The optimal time to inversion $\left(T I_{0}\right)$ is defined visually as the inversion time at which the uninfarcted myocardium (1) is nulled; infarcted myocardium (2)

renal function. Therefore, just before image acquisition, the inversion time is optimised on a per-patient basis using lowresolution scout images at mid-ventricular level with increasing inversion times at intervals of $30 \mathrm{~ms}$, from which the optimal inversion time can be derived [4]. The process is synchronised to the R-wave of the ECG, and mid-diastolic images are acquired every other heart beat during breath-hold [3].

\section{CE-CMR after myocardial infarction}

Myocardial infarction (MI) occurs after coronary occlusion of at least 20-30 min (without sufficient collateral blood supply to the affected myocardium) [5]. In the early phase of myocardial infarction, cellular degradation in the infarcted myocardium results in an increase in the permeability and enlargement of the extravascular space (oedema), and thus, an increased distribution volume for the CMR contrast agent. Later on, due to different wash-in and washout kinetics, myocardial scars retain contrast agents longer than normal myocardium. The net result of both mechanisms is that infarcted myocardium appears bright on $\mathrm{CE} \mathrm{T} 1$ weighted images.

CE in patients with MI generally shows a typical pattern that is related to the perfusion area of the culprit vessel. Myocardial changes (and thus $\mathrm{CE}$ ) of the subendocardium can generally be found which may extend to a transmural distribution in cases with prolonged coronary occlusion. In patients with prior MI, there is a high inter-observer agreement for the assessment of presence and extent of CE [6-8]. In addition, presence, location, and extent of CE correspond well with histology [6-8].

In patients after MI, the assessment of myocardial viability can provide clinically important information to guide further treatment because only viable myocardium may benefit from revascularisation [9]. Generally, a standardised 17-myocardial segment model is used to report the results of viability assessment by CE-CMR (Fig. 2) [10]. In addition, quantification of infarcted tissue helps to prognosticate left ventricular remodelling [11]. In this respect, the transmural extent of infarcted tissue as determined by CE-CMR (Figs. $2 b$ and $3 a, b$ ) has been shown to be a powerful predictor of the contractile response to both medical therapy and myocardial revascularisation [12].

Increasing interest is also laid on the assessment of characteristics of infarcted myocardial tissue as potential predictor of life-threatening ventricular arrhythmias. Recently, a highly significant relation between inferior MI and
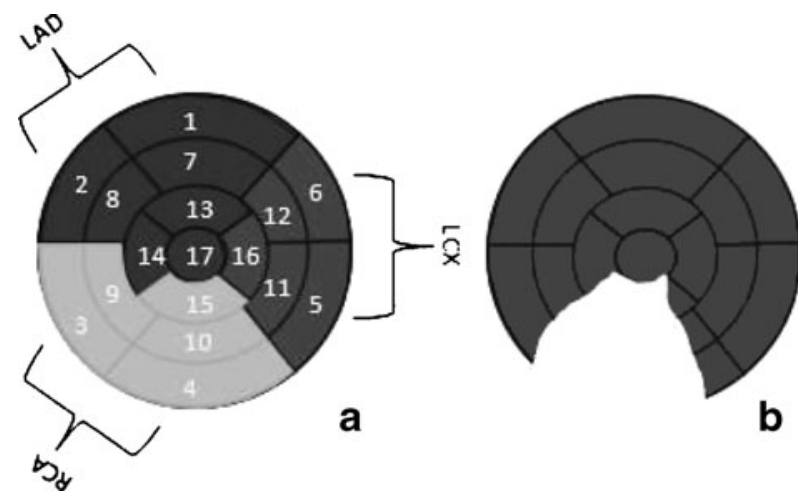

b
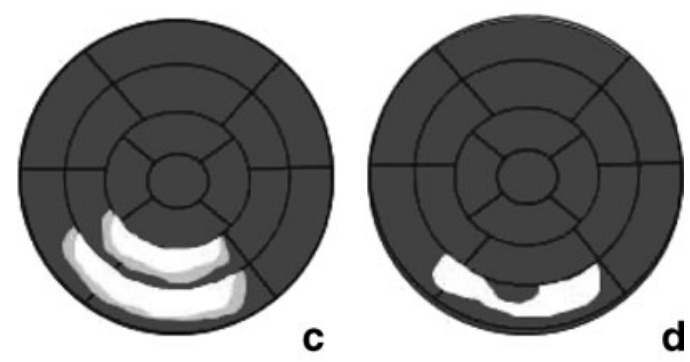

Fig. 2 Bulls eye scheme according to the 17-segmental model, demonstrating $\mathrm{CE}$ characteristics post-myocardial infarction. a Assignment of the 17 segments to one of the three major coronary arteries, with segment $1,2,7,8,13,14$, and 17 corresponding to the left anterior descending coronary artery; segments 3, 4, 9, 10, and 15 corresponding to the right coronary artery when it is dominant; and segments 5, 6, 11, 12, and 16 are assigned to the left circumflex artery. b Transmural inferior MI. c Inferoseptal MI with a core zone (white area) and a peri-infarction zone (grey area). d Inferior MI with microvascular obstruction (black area in myocardial infarction area) 
Fig. 3 CE-CMR patterns postmyocardial infarction. a, b CE short-axis and long-axis view showing transmural inferior infarction (black arrow). c, d Short-axis and long-axis view showing an inferior infarction with microvascular obstruction (white arrow)
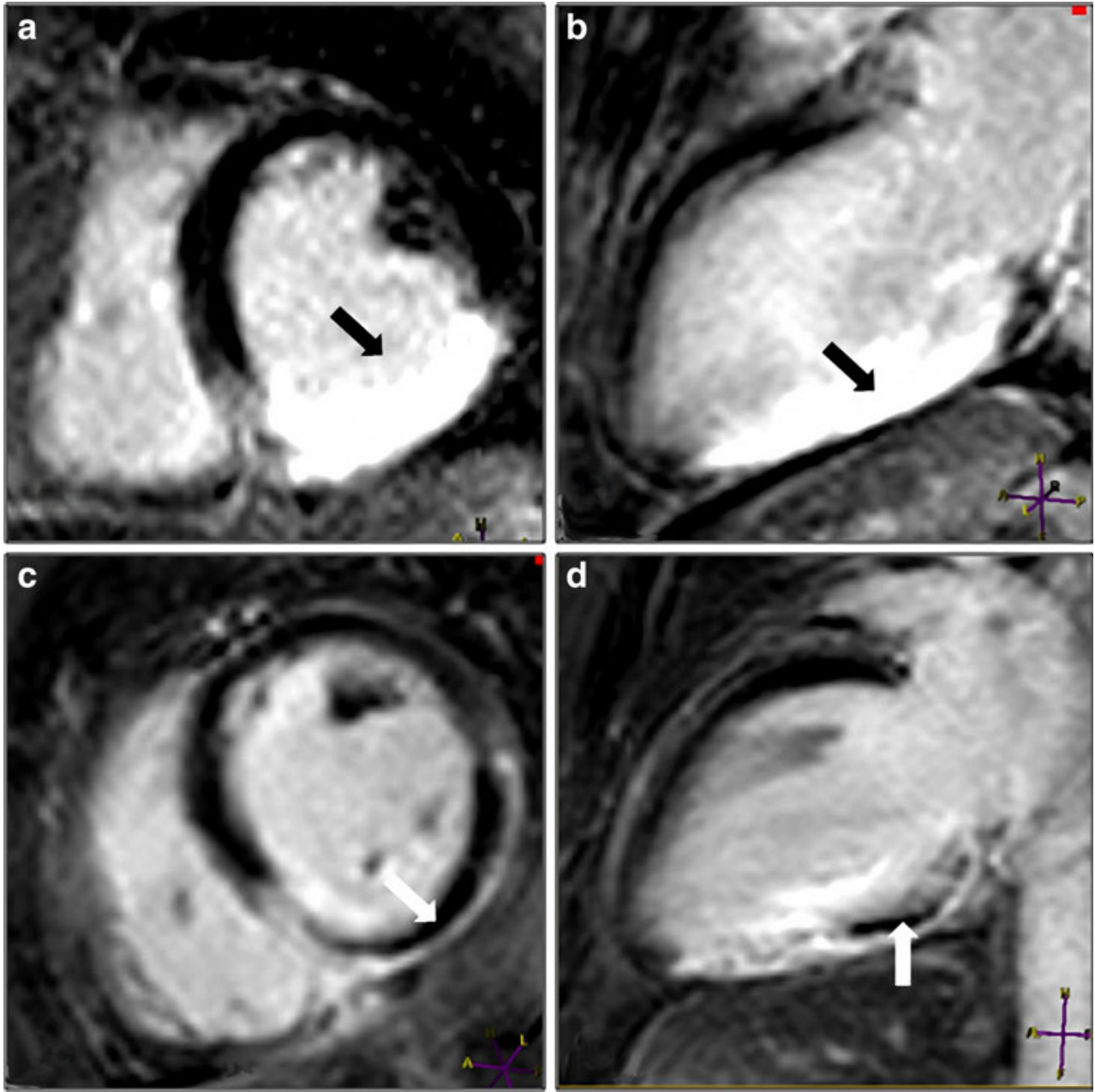

ventricular arrhythmias was observed [13]. Multivariate analysis of data from 91 patients suggested that the heterogeneity of infarcted tissue (also called peri-infarct zone or border zone; Fig. 2c) can be an important predictor of spontaneous ventricular arrhythmias [14].

The area of $\mathrm{CE}$ tends to be larger during the acute phase of MI (first week) and progressively decreases in size during the healing phase (1-4 weeks), until it reaches the state of a healed myocardial infarction (after 4 weeks) [15]. These observations are consistent with the established pathological understanding of remodelling after MI: during the acute phase, there is myocardial oedema which subsequently regresses while the necrotic myocardium is replaced by scar tissue. Experimental studies have revealed that final MI size is strongly influenced by the extent of the oedema in the acute phase, which is also called area at risk (AAR). By combining T2weighted images to visualise myocardial oedema (and thus the AAR) and CE-CMR imaging to visualise scar (the final infarct size), a myocardial salvage index can be calculated by subtracting the infarct size from the AAR [16]. The myocardial salvage index has recently shown to be independently associated with adverse LV remodelling and early ST-segment resolution, and may represent an interesting parameter for the assessment of novel reperfusion strategies in patients with myocardial infarction (trial registration number NL19151.044.07).

In addition, some patients develop microvascular obstruction within the ischaemic myocardial region in the acute phase of an MI [17]. Microvascular obstruction is represented by a dark zone within the infarcted region, usually located in the subendocardium because the contrast medium does not reach this area (Figs. $2 \mathrm{~d}$ and $3 \mathrm{c}$, d). Its presence is associated with greater LV remodelling and inferior clinical outcome $[18,19]$.

\section{CE-CMR in nonischaemic cardiomyopathy}

Hypertrophic cardiomyopathy

Hypertrophic cardiomyopathy (CMP) is a primary myocardial disease characterised by focal (mostly septal) or diffuse LV wall thickening (with or without LV outflow obstruction). 
Myofibrillar hypertrophy and disarray [20] and myocardial fibrosis have been described histologically [3]. Inadequate capillary density and intimal hyperplasia of intramural coronary arteries, which were also seen in such patients, may contribute to myocardial ischaemia [3]. There are predilection patterns of $\mathrm{CE}$ in patients with hypertrophic CMP: more than $80 \%$ of patients exhibit patchy fibrosis at the right ventricular insertion points and in the anteroseptal wall in the region of characteristic septal thickening (Figs. 4a and 5a, b) [21, 22]. Myocardial fibrosis, however, is also located in non-hypertrophic segments [23, 24]. As the amount of CE in hypertrophic CMP often corresponds with functional parameters and the frequency of cardiac events, CE-CMR may potentially be useful for risk stratification [25]. However, data regarding the prognostic value of these findings remain scarce; therefore, further research is warranted [26].

Presence and extent of $\mathrm{CE}$ following percutaneous transseptal myocardial ablation (by alcohol injection) for the treatment of significant LV outflow tract obstruction indicate the location and extent of therapeutic myocardial tissue destruction [27].

Idiopathic dilated cardiomyopathy

Idiopathic dilated CMP is characterised by dilation and impaired contractility of the left ventricle or both ventricles in the absence of abnormal loading conditions (e.g., arterial hypertension, valvular disease), and/or a CMP with a distinct cause (e.g., ischaemic heart disease; peripartum cardiomyopathy; toxin-, chemotherapy-, or tachycardiainduced cardiomyopathy; certain endocrinopathies) [1]. Histology is nonspecific, and a variety of myocardial tissue alterations may occur or coexist, including myocyte hypertrophy and segmental or diffuse interstitial fibrosis. Current CE-CMR techniques are unlikely to detect diffuse fibrosis due to limited voxel resolution [28]. Myocardial fibrosis in idiopathic dilated CMP is mostly seen in the LV midwall with septal predominance and a linear pattern (Figs. 4b and 5c, d); however, it has occasionally been described at subendocardial and subepicardial locations with a more patchy pattern [23]. Of note, in various studies, the prevalence of myocardial fibrosis varied from $13 \%$ to $62 \%[29,30]$. It has been suggested that the degree of $\mathrm{CE}$ may correlate with functional impairment of the LV [31]. There are preliminary data demonstrating that the presence of $\mathrm{CE}$ is associated with an unfavourable clinical outcome and may be a predictor of sudden death in patients with idiopathic dilated CMP [29, 32, 33].

\section{Arrhythmogenic right ventricular cardiomyopathy}

The arrhythmogenic right ventricular cardiomyopathy (ARVC) is characterised by structural and functional abnormalities, with progressive fibrous and fatty infiltration involving variable regions of the right and left ventricular myocardium. This process finally leads to progressive RV failure and ventricular tachyarrhythmia [23]. Diagnosis of this condition remains a challenge, with nonspecific abnormalities on echocardiographic and angiographic examinations. Endomyocardial biopsy has a low sensitivity, as samples are usually taken from the septum, a region that is infrequently involved [23]. Information from CE-CMR may help to guide targeted endomyocardial biopsies. Predilection patterns with midwall $\mathrm{CE}$ are found in the basal anterior region (Fig. 4c) and/or the RV outflow tract. These patterns of fibrosis correlate with fibro-fatty replacement of the myocardium at histological assessment and predict induction of ventricular tachycardia during electrophysiological studies. As the presence of ARVC cannot be ruled out based on CMR findings alone, standardised guidelines have been proposed which define major and minor criteria, including morphological, histological, electrocardiographic, functional, and genetic characteristics [34, 35].
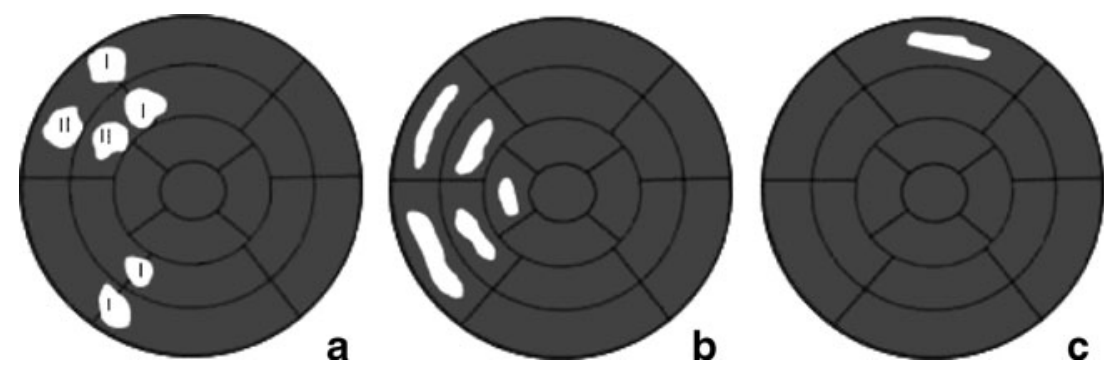

Fig. 4 Bulls eye scheme according to the 17-segmental model, demonstrating typical CE patterns in nonischaemic cardiomyopathies. a Hypertrophic cardiomyopathy with predilection CE (i.e., fibrosis) pattern at the right ventricular insertion points $(I)$ and anteroseptal (II). Myocardial fibrosis is also located in non-hypertrophic segments. b
Idiopathic dilated cardiomyopathy with CE predominantly located in the midwall with septal predominance. c Arrhythmogenic right ventricular cardiomyopathy with midwall $\mathrm{CE}$ found in the basal anterior region. $\mathrm{CE}$ is also found in the right ventricular outflow tract 
Fig. 5 CE-CMR patterns in nonischaemic cardiomyopathies. a, b CE short-axis and long-axis view showing hypertrophic cardiomyopathy with patchy $\mathrm{CE}$ septal and in the right ventricular insertion points (black arrow). c, d Short-axis and long-axis view showing dilated cardiomyopathy with $\mathrm{CE}$ having septal midwall predominance (white arrow)
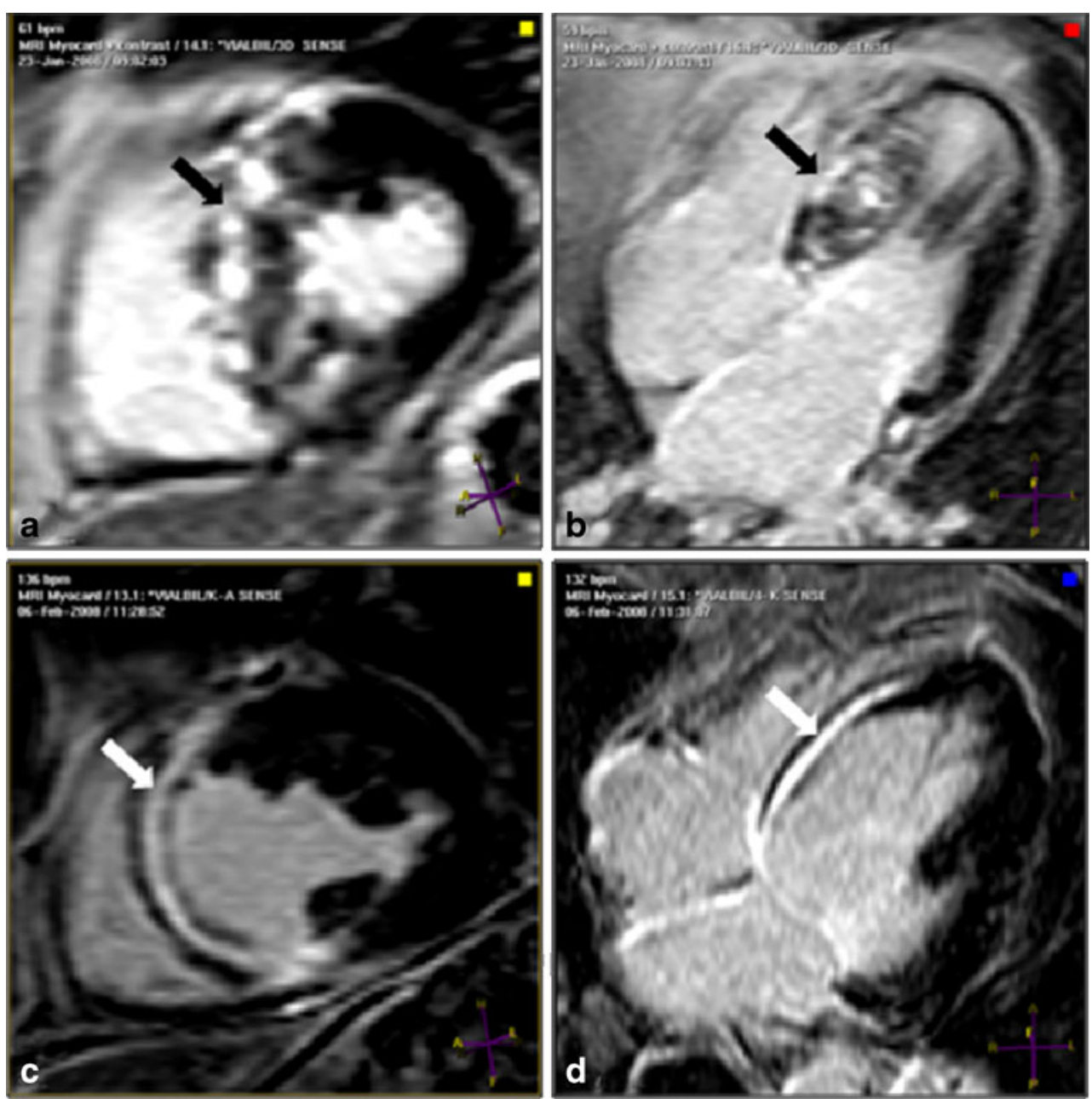

\section{CE-CMR in myocarditis}

\section{Myocarditis}

Myocarditis is an acute or chronic inflammatory disease of the myocardium, which can be caused by viruses or initiated by post-infectious immune or primarily organspecific autoimmune responses [36]. Patients generally recover or infrequently develop dilated CMP, sometimes even with life-threatening complications including severe heart failure and malignant arrhythmias [37]. Diagnosis of myocarditis is challenging because of a diverse clinical presentation and a limited sensitivity of endomyocardial biopsies, but may be facilitated by use of CE-CMR or myocardial global relative enhancement CMR [38, 39]. Presence of CE has been reported in $44-95 \%$ of patients with myocarditis, [40, 41] indicating areas of myocardial damage with a sensitivity of $100 \%$ and a specificity of $90 \%$ (compared with histopathology) [40]. In acute myocarditis, $\mathrm{CE}$ is frequently located in the lateral wall, originating from the epicardium. The subendocardium is generally not involved, with the exception of eosinophilic myocarditis which frequently involves the endomyocardium (Fig. 6a) [23, 42]. In chronic myocarditis, besides an increased oedema on T2-weighted imaging, an increased global relative enhancement is a common finding as confirmed in immunohistological analyses [38]. CE-CMR identified areas of myocardial damage in $70 \%$ of patients with biopsyproven chronic myocarditis and showed a predilection pattern (LV midwall and/or subepicardial). CE may also help to guide targeted endomyocardial biopsies. In myocarditis, CE may provide additional information that could help to differentiate between viral origins; in the majority of parvovirus B19 patients, CE is found in the lateral free wall, while in patients with HHV6 myocarditis, $\mathrm{CE}$ frequently involves the interventricular septal midwall [43]. In addition, we recently studied a limited number of patients with chronic fatigue syndrome and concomitant Ebstein Barr virus or cytomegalovirus myocarditis who showed a certain predilection of the septal region in the presence of CE. Inflammatory activity on T2-weighted imaging and myocardial fibrosis on CECMR may have relevant prognostic implications in acute and chronic myocarditis and may ultimately serve as a tool 

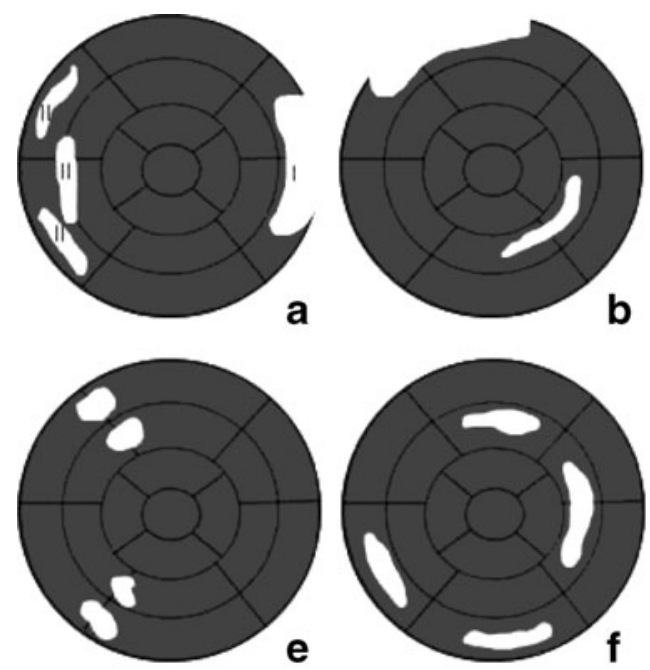

Fig. 6 Bulls eye scheme according to the 17-segmental model, demonstrating typical CE patterns in myocarditis and cardiac involvement of other diseases. a Myocarditis with $\mathrm{CE}$ frequently located in the lateral wall originating from the epicardium $(I)$. $\mathrm{CE}$ patterns in myocarditis differ according to viral origin, with parvovirus B19 having $\mathrm{CE}$ in the lateral free wall (I), HHV6 having $\mathrm{CE}$ frequently in the interventricular septal midwall (II), and chronic fatigue syndrome myocarditis having CE anteroseptal and inferoseptal (III). b Sarcoidosis with CE midwall or epicardial; however,

to triage patients [44]. In addition, cardiac function and regression of myocardial changes can be well observed with CMR.

\section{CE-CMR in intracardiac masses}

Intracardiac masses can be characterised as thrombi, primary benign or malignant tumours or metastases of the heart. Along with cine gradient echo and both T1-weighted and T2-weighted CMR images, CE-CMR imaging (with its capability of characterising tissue) can provide important additional information in the evaluation of intracardiac masses. A correct suggestion for the aetiology of intracardiac masses has been reported in $75 \%$ of cases by CMR, compared with $29 \%$ by echocardiography [45]. Cardiac thrombi are overall the most frequent intracardiac masses $[46,47]$. Myxomas, the most common benign tumours of the heart, have a high signal intensity on T2-weighted images (due to a high extracellular water content) and will generally enhance heterogeneously with CE (reflecting varying components of myxoid, haemorrhagic, cystic, calcified, and fibrous tissue); thrombi - on the other hand - will generally not enhance [48, 49]. Features of malignant tumours at CMR are invasive spreading, involvement of the right side of the heart, heterogeneous tissue with low and high signal intensities on T1 images, a diameter greater than

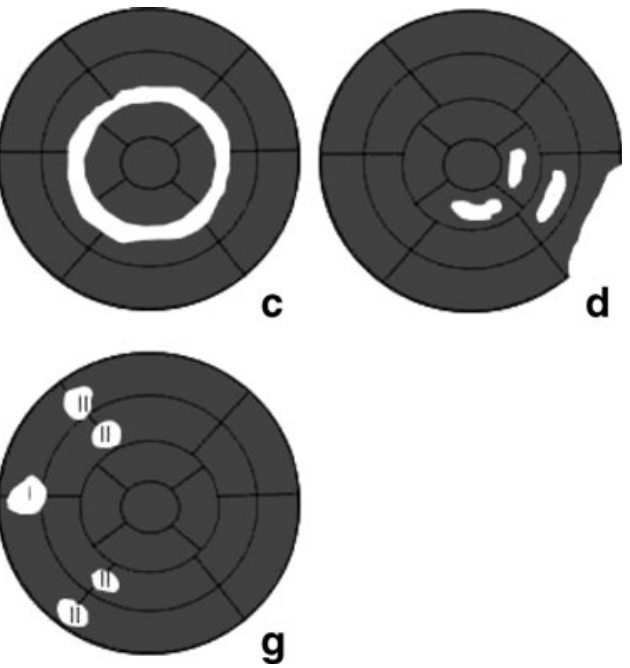

subendocardial or transmural CE may be observed. c Amyloidosis, usually with a global diffuse CE pattern, frequently involving the subendocardium. d Chagas' disease with CE epicardial or midwall, with a predilection pattern inferolateral. e Pulmonary hypertension with $\mathrm{CE}$ involving the right ventricular insertion points and the interventricular septum. f Muscular dystrophy with CE observed in the midwall. g Chloroquine-induced cardiomyopathy with hypertrophy and accompanying $\mathrm{CE}$ in the basal septum $(I)$ and the right ventricular insertion points $(I)$

$5 \mathrm{~cm}$, and/or the presence of increased CE (as a result of an increased vascularisation).

\section{CE-CMR in systemic diseases}

\section{Sarcoidosis}

Cardiac involvement in sarcoidosis, a multisystem granulomatous disorder of unknown aetiology, is clinically often asymptomatic (95\%) while autopsy revealed cardiac manifestation in up to $60 \%$ [50]. Advanced sarcoidosis leads to septal thinning, systolic and diastolic dysfunction, and pericardial effusion, which can easily be detected with echocardiography [4]. Early sarcoidosis, however, is more challenging to diagnose, and CMR can be very useful in this context. During the acute stage of this disease, regions of active inflammation and oedema are visible on T2weighted images as areas of increased signal intensity. During the chronic stage, CE will typically appear as a midwall or epicardial nonischaemic pattern (Fig. 6b), but occasionally, subendocardial or transmural $\mathrm{CE}$ may be observed, mimicking a pattern of post-MI. CE has been found in $50 \%$ of all patients diagnosed with sarcoidosis [51]. CE-CMR may also be useful to evaluate the response to therapy. In a CMR study of 16 patients with sarcoidosis, CE was markedly diminished 1 month after the initiation of 
steroid therapy [51]. CE in sarcoidosis patients may be associated with future adverse events (including cardiac death), but confirmation in larger patient cohorts is required [50].

\section{Amyloidosis}

Both primary and secondary amyloidosis are characterised by extracellular deposition of fibrillar proteins, [1] which may lead to restrictive cardiomyopathy with an initially preserved systolic LV function [52]. In cardiac amyloidosis, $\mathrm{CE}$ is commonly found as a result of the expansion of interstitial space and some endomyocardial fibrosis, [53] leading to a usually global and diffuse CE pattern [4]. Although the subendocardium is commonly involved (as in ischaemic heart disease), the distribution of $\mathrm{CE}$ is not related to a particular coronary perfusion area (Fig. 6c) [4].

\section{Chagas' disease}

The parasitic protozoan Trypanosoma cruzi causes Chagas' disease, which is endemic in the Latin American region [4]. During chronic disease, the heart is the most frequently affected organ, and patients present with refractory heart failure, disorders of the conduction system, or ventricular tachycardia [3,54]. The fundamental pathological processes include an inflammatory response, cellular damage with a broad variation of intensity (minimal alterations up to extensive necrosis), and fibrosis [55]. Early cardiac involvement may be detected by CE-CMR before the onset of symptoms [56]. CE is often seen epicardially or in the LV midwall with an inferolateral predilection pattern (Fig. 6d), but other regions - including the apex - may also sometimes be affected [4].

\section{Pulmonary hypertension}

Pulmonary arterial hypertension, both primary and secondary, is characterised by an increased pulmonary vascular resistance that results in pressure overload on the right ventricle [57]. Cine CMR permits accurate assessment of RV mass and volumes which is often difficult to accomplish with other imaging modalities [3]. Myocardial CE is frequently observed in patients with severe symptomatic pulmonary artery hypertension with predilection patterns involving both right ventricular septal insertion points and the interventricular septum (Fig. 6e). CE in the interventricular septum was found to be associated with septal bowing (on cine CMR), and the extent of $\mathrm{CE}$ correlated inversely with right ventricular systolic function [58].
Muscular dystrophy

Both Becker and Duchenne muscular dystrophies are progressive X chromosome-linked recessive neuromuscular diseases with myocardial involvement in up to $72 \%$ of patients showing a mildly reduced LV function up to severe LV impairment and dilated CMP. Cardiac myocyte dystrophin deficiency leads to necrosis causing replacement of damaged myocardium by connective tissue and fat in both ventricles. On CE-CMR, hyperenhancement is predominantly seen in LV midwall (Fig. 6f) and has been described in $73-100 \%$ of patients [59]. Early diagnosis of myocardial involvement as assessed with CE-CMR may permit an earlier treatment of heart failure which could increase life expectancy.

Chloroquine-induced cardiomyopathy

Chloroquine-induced cardiomyopathy is a rare iatrogenic disease that is associated with long-term intake of chloroquine, which is most frequently prescribed for treatment of rheumatoid arthritis and malaria prophylaxis [60]. This CMP is characterised by ineffective lysosomal metabolism because of an increase in $\mathrm{pH}$ that leads to accumulation of lysosomal glycosphingolipids and finally thickening of cardiac walls [61]. The time interval between the start of chloroquine therapy and disease manifestation varies greatly, ranging from several months to more than 20 years [60]. CMR may demonstrate the presence of LV hypertrophy with accompanying areas of CE in the basal septum and at the insertion point of the right ventricle (Fig. 6g).

Fabry disease, an $\mathrm{X}$ chromosome-linked lysosomal storage disease caused by a deficient activity of the enzyme $\alpha$-galactosidase $\mathrm{A}$, can also result in the accumulation of glycosphingolipids in multiple organs, including the heart $[61,62]$. Fabry disease cardiomyopathy should therefore always be considered in the differential diagnosis of 'idiopathic' LV hypertrophy (in the absence of arterial hypertension or valvular disease).

\section{Future developments}

Several new strategies for the treatment of the various forms of cardiomyopathy are currently under development, including the transplantation of primitive cell types (e.g., stem cells or myoblasts) into damaged myocardium in an attempt to promote trans-differentiation into functional myocardial cells. CE-CMR imaging can be used to monitor such studies and to evaluate the results of novel therapeutic strategies such as direct injection of primitive cell types into segments with transmural infarction [63]. In animal models, 
mesenchymal stem cells have been labelled with iron-based contrast agents to examine the process of 'homing' of such cells in the myocardium [64, 65, 66].

Recently, 3.0-T CMR imaging with a 3D inversionrecovery gradient-echo sequence was compared with standard 2D imaging. The 3D technique showed superior spatial image resolution, shorter image acquisition time, preserved contrast-to-noise ratio, and similar intra- and interobserver variabilities (compared with the 2D approach), which could improve the clinical utility of CE-CMR in the future [67]. At higher heart rates, though, motion artefacts can be seen.

\section{Conclusion}

Besides its use in patients with ischaemic heart disease, CECMR is increasingly used to establish the diagnosis, monitor therapy, and obtain prognostic information in a variety of cardiac diseases. While CE-CMR can provide valuable information, there is considerable overlap in $\mathrm{CE}$ patterns between different cardiac diseases. For that reason, CE-CMR findings should be considered in the light of the clinical history and presentation as well as findings obtained from other diagnostic modalities.

Open Access This article is distributed under the terms of the Creative Commons Attribution Noncommercial License which permits any noncommercial use, distribution, and reproduction in any medium, provided the original author(s) and source are credited.

\section{References}

1. Mueller GC, Attili A. Cardiomyopathy: magnetic resonance imaging evaluation. Semin Roentgenol. 2008;43(3):204-22.

2. Vogel-Claussen J, Rochitte $\mathrm{CE}, \mathrm{Wu} \mathrm{KC}$, et al. Delayed enhancement MR imaging: utility in myocardial assessment. Radiographics. 2006;26(3):795-810.

3. Marcu CB, Nijveldt R, Beek AM, et al. Delayed contrast enhancement magnetic resonance imaging for the assessment of cardiac disease. Heart Lung Circ. 2007;16(2):70-8.

4. Jackson E, Bellenger N, Seddon M, et al. Ischaemic and nonischaemic cardiomyopathies-cardiac MRI appearances with delayed enhancement. Clin Radiol. 2007;62(5):395-403.

5. Edelman RR. Contrast-enhanced MR imaging of the heart: overview of the literature. Radiology. 2004;232(3):653-68.

6. Amado LC, Gerber BL, Gupta SN, et al. Accurate and objective infarct sizing by contrast-enhanced magnetic resonance imaging in a canine myocardial infarction model. J Am Coll Cardiol. 2004;44(12):2383-9.

7. Hsu LY, Natanzon A, Kellman P, et al. Quantitative myocardial infarction on delayed enhancement MRI. Part I: animal validation of an automated feature analysis and combined thresholding infarct sizing algorithm. J Magn Reson Imaging. 2006;23(3):298308.
8. Bello D, Fieno DS, Kim RJ, et al. Infarct morphology identifies patients with substrate for sustained ventricular tachycardia. J Am Coll Cardiol. 2005;45(7):1104-8.

9. Kim RJ, Wu E, Rafael A, et al. The use of contrast-enhanced magnetic resonance imaging to identify reversible myocardial dysfunction. N Engl J Med. 2000;343(20):1445-53.

10. Cerqueira MD, Weissman NJ, Dilsizian V, et al. Standardized myocardial segmentation and nomenclature for tomographic imaging of the heart: a statement for healthcare professionals from the Cardiac Imaging Committee of the Council on Clinical Cardiology of the American Heart Association. Circulation. 2002;105(4):539-42.

11. Orn S, Manhenke C, Anand IS, et al. Effect of left ventricular scar size, location, and transmurality on left ventricular remodeling with healed myocardial infarction. Am J Cardiol. 2007;99 (8):1109-14.

12. Weinsaft JW, Klem I, Judd RM. MRI for the assessment of myocardial viability. Cardiol Clin. 2007;25(1):35-56. v.

13. Pascale P, Schlaepfer J, Oddo M, et al. Ventricular arrhythmia in coronary artery disease: limits of a risk stratification strategy based on the ejection fraction alone and impact of infarct localization. Europace. 2009;11(12):1639-46.

14. Roes SD, Borleffs C, van der Geest RJ, et al. Infarct tissue heterogeneity assessed with contrast-enhanced MRI predicts spontaneous ventricular arrhythmia in patients with ischemic cardiomyopathy and implantable cardioverter-defibrillator. Circ Cardiovasc Imaging. 2009;2(3):183.

15. Thygesen K, Alpert JS, White HD. Universal definition of myocardial infarction. Eur Heart J. 2007;28(20):2525-38.

16. Aletras AH, Tilak GS, Natanzon A, et al. Retrospective determination of the area at risk for reperfused acute myocardial infarction with $\mathrm{T} 2$-weighted cardiac magnetic resonance imaging: histopathological and displacement encoding with stimulated echoes (DENSE) functional validations. Circulation. 2006;113 (15):1865-70.

17. Masci PG, Ganame J, Strata E, et al. Myocardial salvage by CMR correlates with LV remodeling and early ST-segment resolution in acute myocardial infarction. JACC Cardiovasc Imaging. 2010;3 (1):45-51.

18. Nijveldt R, Beek AM, Hirsch A, et al. Functional recovery after acute myocardial infarction: comparison between angiography, electrocardiography, and cardiovascular magnetic resonance measures of microvascular injury. J Am Coll Cardiol. 2008;52 (3):181-9.

19. Nijveldt R, Beek AM, Hirsch A, et al. 'No-reflow' after acute myocardial infarction: direct visualisation of microvascular obstruction by gadolinium-enhanced CMR. Neth Heart J. 2008;16(5):179-81.

20. Christiaans I, Nannenberg EA, Dooijes D, et al. Founder mutations in hypertrophic cardiomyopathy patients in the Netherlands. Neth Heart J. 2010;18(5):248-54.

21. Moon JC, McKenna WJ, McCrohon JA, et al. Toward clinical risk assessment in hypertrophic cardiomyopathy with gadolinium cardiovascular magnetic resonance. J Am Coll Cardiol. 2003;41 (9):1561-7.

22. Choudhury L, Mahrholdt H, Wagner A, et al. Myocardial scarring in asymptomatic or mildly symptomatic patients with hypertrophic cardiomyopathy. J Am Coll Cardiol. 2002;40 (12):2156-64.

23. Bohl S, Wassmuth R, Abdel-Aty H, et al. Delayed enhancement cardiac magnetic resonance imaging reveals typical patterns of myocardial injury in patients with various forms of non-ischemic heart disease. Int J Cardiovasc Imaging. 2008;24(6):597-607.

24. van der Wall EE, Siebelink HM, Bax JJ. Evaluation of hypertrophic cardiomyopathy: new horizons for CMR? Neth Heart J. 2010;18(3):116-7. 
25. Rubinshtein R, Glockner JF, Ommen SR, et al. Characteristics and clinical significance of late gadolinium enhancement by contrast-enhanced magnetic resonance imaging in patients with hypertrophic cardiomyopathy. Circ Heart Fail. 2010;3(1):518.

26. Germans T, Nijveldt R, Brouwer WP, et al. The role of cardiac magnetic resonance imaging in differentiating the underlying causes of left ventricular hypertrophy. Neth Heart J. 2010;18 (3): 135-43.

27. Sievers B, Moon JC, Pennell DJ. Images in cardiovascular medicine. Magnetic resonance contrast enhancement of iatrogenic septal myocardial infarction in hypertrophic cardiomyopathy. Circulation. 2002;105(8):1018.

28. Vohringer M, Mahrholdt H, Yilmaz A, et al. Significance of late gadolinium enhancement in cardiovascular magnetic resonance imaging (CMR). Herz. 2007;32(2):129-37.

29. Yokokawa M, Tada H, Koyama K, et al. The characteristics and distribution of the scar tissue predict ventricular tachycardia in patients with advanced heart failure. Pacing Clin Electrophysiol. 2009;32(3):314-22.

30. Isbell DC, Kramer CM. The evolving role of cardiovascular magnetic resonance imaging in nonischemic cardiomyopathy. Semin Ultrasound CT MR. 2006;27(1):20-31.

31. Koito H, Suzuki J, Ohkubo N, et al. Gadolinium-diethylenetriamine pentaacetic acid enhanced magnetic resonance imaging of dilated cardiomyopathy: clinical significance of abnormally high signal intensity of left ventricular myocardium. J Cardiol. 1996;28(1):41-9.

32. Yokokawa M, Tada H, Koyama K, et al. Nontransmural scar detected by magnetic resonance imaging and origin of ventricular tachycardia in structural heart disease. Pacing Clin Electrophysiol. 2009;32 Suppl 1:S52-6.

33. Assomull RG, Prasad SK, Lyne J, et al. Cardiovascular magnetic resonance, fibrosis, and prognosis in dilated cardiomyopathy. J Am Coll Cardiol. 2006;48(10):1977-85.

34. McKenna WJ, Thiene G, Nava A, et al. Diagnosis of arrhythmogenic right ventricular dysplasia/cardiomyopathy. Task Force of the Working Group Myocardial and Pericardial Disease of the European Society of Cardiology and of the Scientific Council on Cardiomyopathies of the International Society and Federation of Cardiology. Br Heart J. 1994;71 (3):215-8.

35. Marcus FI, McKenna WJ, Sherrill D, et al. Diagnosis of arrhythmogenic right ventricular cardiomyopathy/dysplasia: proposed modification of the Task Force Criteria. Eur Heart J. 2010;31(7):806-14.

36. Caforio AL, Mahon NJ, Tona F, et al. Circulating cardiac autoantibodies in dilated cardiomyopathy and myocarditis: pathogenetic and clinical significance. Eur J Heart Fail. 2002;4 (4):411-7.

37. Zagrosek A, Wassmuth R, Abdel-Aty H, et al. Relation between myocardial edema and myocardial mass during the acute and convalescent phase of myocarditis - a CMR study. J Cardiovasc Magn Reson. 2008;10(1):19.

38. Gutberlet M, Spors B, Thoma T, et al. Suspected chronic myocarditis at cardiac MR: diagnostic accuracy and association with immunohistologically detected inflammation and viral persistence. Radiology. 2008;246(2):401-9.

39. Abdel-Aty H, Boye P, Zagrosek A, et al. Diagnostic performance of cardiovascular magnetic resonance in patients with suspected acute myocarditis: comparison of different approaches. J Am Coll Cardiol. 2005;45(11):1815-22.

40. Mahrholdt H, Goedecke C, Wagner A, et al. Cardiovascular magnetic resonance assessment of human myocarditis: a comparison to histology and molecular pathology. Circulation. 2004;109 (10):1250-8.
41. Mahrholdt H, Wagner A, Deluigi CC, et al. Presentation, patterns of myocardial damage, and clinical course of viral myocarditis. Circulation. 2006;114(15):1581-90.

42. Deb K, Djavidani B, Buchner S, et al. Time course of eosinophilic myocarditis visualized by CMR. J Cardiovasc Magn Reson. 2008;10(1):21.

43. Yelgec NS, Dymarkowski S, Ganame J, et al. Value of MRI in patients with a clinical suspicion of acute myocarditis. Eur Radiol. 2007;17(9):2211-7.

44. Olimulder MA, van Es J, Galjee MA. The importance of cardiac MRI as a diagnostic tool in viral myocarditis-induced cardiomyopathy. Neth Heart J. 2009;17(12):481-6.

45. Gulati G, Sharma S, Kothari SS, et al. Comparison of echo and MRI in the imaging evaluation of intracardiac masses. Cardiovasc Interv Radiol. 2004;27(5):459-69.

46. Baur LH. Intracardiac thrombi: diagnostic accuracy needs to be improved! Int J Cardiovasc Imaging. 2007;23(4):489-90.

47. Kim DH, Choi SI, Choi JA, et al. Various findings of cardiac thrombi on MDCT and MRI. J Comput Assist Tomogr. 2006;30 (4):572-7.

48. Sparrow PJ, Kurian JB, Jones TR, et al. MR imaging of cardiac tumors. Radiographics. 2005;25(5):1255-76.

49. Butany J, Nair V, Naseemuddin A, et al. Cardiac tumours: diagnosis and management. Lancet Oncol. 2005;6(4):219-28.

50. Patel MR, Cawley PJ, Heitner JF, et al. Detection of myocardial damage in patients with sarcoidosis. Circulation. 2009;120 (20):1969-77.

51. Shimada T, Shimada K, Sakane T, et al. Diagnosis of cardiac sarcoidosis and evaluation of the effects of steroid therapy by gadolinium-DTPA-enhanced magnetic resonance imaging. Am J Med. 2001;110(7):520-7.

52. Wynne J, Braunwald E. Restrictive and infiltrative cardiomyopathies. In: Zipes DP, Libby P, Bonow RO, Braunwald E, editors. Braunwald's heart disease: a textbook of cardiovascular medicine. Philadelphia: Elsevier Saunders; 2005. p. 1682-92.

53. Maceira AM, Prasad SK, Hawkins PN, et al. Cardiovascular magnetic resonance and prognosis in cardiac amyloidosis. J Cardiovasc Magn Reson. 2008;10(1):54.

54. Rochitte CE, Oliveira PF, Andrade JM, et al. Myocardial delayed enhancement by magnetic resonance imaging in patients with Chagas' disease: a marker of disease severity. J Am Coll Cardiol. 2005;46(8):1553-8.

55. Rochitte CE, Nacif MS, de Oliveira Junior AC, et al. Cardiac magnetic resonance in Chagas' disease. Artif Organs. 2007;31 (4):259-67.

56. Rochitte CE, Olivera PF, Joalbo M. Chagas disease is characterised by specific pattern and location of myocardial delayed enhancement MRI. J Cardiovasc Magn Reson. 2003;5:115. Ref Type: Generic.

57. Kovacs G, Reiter G, Reiter U, et al. The emerging role of magnetic resonance imaging in the diagnosis and management of pulmonary hypertension. Respiration. 2008;76(4):458-70.

58. Blyth KG, Groenning BA, Martin TN, et al. Contrast enhancedcardiovascular magnetic resonance imaging in patients with pulmonary hypertension. Eur Heart J. 2005;26(19):1993-9.

59. Yilmaz A, Gdynia HJ, Baccouche H, et al. Cardiac involvement in patients with Becker muscular dystrophy: new diagnostic and pathophysiological insights by a CMR approach. J Cardiovasc Magn Reson. 2008;10(1):50.

60. Reffelmann T, Naami A, Spuentrup E, et al. Images in cardiovascular medicine. Contrast-enhanced magnetic resonance imaging of a patient with chloroquine-induced cardiomyopathy confirmed by endomyocardial biopsy. Circulation. 2006;114(8): e357-8.

61. Pieroni M, Bellocci F, Crea F. Letter by Pieroni et al regarding article, "Contrast-enhanced magnetic resonance imaging of a 
patient with chloroquine-induced cardiomyopathy confirmed by endomyocardial biopsy". Circulation. 2007;115(5):e67.

62. Gange CA, Link MS, Maron MS. Utility of cardiovascular magnetic resonance in the diagnosis of Anderson-Fabry disease. Circulation. 2009;120(13):e96-7.

63. Orlic D, Hill JM, Arai AE. Stem cells for myocardial regeneration. Circ Res. 2002;91(12):1092-102.

64. Hill JM, Dick AJ, Raman VK, et al. Serial cardiac magnetic resonance imaging of injected mesenchymal stem cells. Circulation. 2003;108(8):1009-14.
65. Kraitchman DL, Heldman AW, Atalar E, et al. In vivo magnetic resonance imaging of mesenchymal stem cells in myocardial infarction. Circulation. 2003;107(18):2290-3.

66. Garot J, Unterseeh T, Teiger E, et al. Magnetic resonance imaging of targeted catheter-based implantation of myogenic precursor cells into infarcted left ventricular myocardium. J Am Coll Cardiol. 2003;41(10):1841-6.

67. Bauner KU, Muehling O, Theisen D, et al. Assessment of myocardial viability with $3 \mathrm{D}$ MRI at $3 \mathrm{~T}$. AJR Am J Roentgenol. 2009;192(6): 1645-50. 